
stratégies de formation

\title{
Espaces énonciatifs du journal télévisé : un retour de l'énoncé?
}

\section{Eliséo Véron}

\section{(2) OpenEdition}

Journals

Édition électronique

URL : http://journals.openedition.org/edc/2847

DOI : 10.4000/edc. 2847

ISSN : 2101-0366

Éditeur

Université Lille-3

Édition imprimée

Date de publication : 1 mai 1989

Pagination : 67-72

ISSN : 1270-6841

Référence électronique

Eliséo Véron, «Espaces énonciatifs du journal télévisé : un retour de l'énoncé ? 》, Études de communication [En ligne], 10 | 1989, mis en ligne le 11 février 2012, consulté le 20 avril 2019. URL http://journals.openedition.org/edc/2847 ; DOI : 10.4000/edc.2847

Ce document a été généré automatiquement le 20 avril 2019

(c) Tous droits réservés 


\title{
Espaces énonciatifs du journal télévisé : un retour de l'énoncé ?
}

\author{
Eliséo Véron
}

1 J'aborderai ici, d'une manière forcément schématique, certains aspects de l'évolution du langage audiovisuel des informations télévisées en France. Étant entendu qu'en l'occurrence, il conviendra d'avoir surtout présent à l'esprit le représentant le plus important de ce genre (en termes d'audience, et partant en termes de son rôle dans la construction de «l'image de marque » d'une chaîne) à savoir le journal télévisé de vingt heures.

2 En préambule, je rappellerai les grandes lignes de l'évolution historique des journaux télévisés. Des travaux ont déjà été publiés sur ce thème. Je ferai ce rappel du point de vue qui m'intéresse : l'évolution des modalités de discours.

Dans une première période, que Brusini et James ont décrit comme celle du « journalisme de reportage " à la télévision ${ }^{1}$, l'objectivité de l'information s'est construite sur ce que l'on pourrait appeler le registre du neutre, qui consistait à annuler le plus possible des niveaux de discours qui ne se sont mis à fonctionner que beaucoup plus tard. A cette période correspond ce que j'ai appelé, il y a quelques années, le "présentateur ventriloque ${ }^{2}$. La légitimité de l'information se fondait en fait sur une stratégie de l'oralité, étrangère à l'image audiovisuelle elle-même, plus précisément étrangère à l'espace-temps de l'image. Il ne faut pas oublier à cet égard, que dans beaucoup de pays les premiers présentateurs de journaux télévisés ont été des journalistes de radio passés à la télévision.

4 Le présentateur regardant la caméra (c'est-à-dire les téléspectateurs) a été la figure caractéristique du journal télévisé depuis son origine, marquant ainsi la spécificité du nouveau type de discours vis-à-vis de son ancêtre, les "actualités cinématographiques ", qui avaient joué un rôle informatif extrêmement important, en particulier pendant la deuxième guerre mondiale. Le présentateur était déjà là comme marqueur d'identification typologique (ouverture et fermeture du discours), mais il fonctionnait au degré zéro de l'expression. L'essentiel du journal était encore proche de son ancêtre : construit sous la forme d'un 'montage de «sujets " successifs, commentés par le présentateur en voix off. 
Les manoeuvres énonciatives dans l'espace-temps de l'image (gestualité et contexte immédiat) étant réduites au minimum, la crédibilité de l'information racontée reposait entièrement sur le contenu des propos tenus, sur la matière verbale. La seule condition d'énonciation était la production d'un certain sérieux, associé à cette neutralité élocutive.

A partir de ce modèle initial, l'évolution du journal télévisé a consisté, au fil des ans, à «mettre en mouvement» les potentialités énonciatives contenues dans cette image audiovisuelle timidement proposée au départ : un présentateur qui nous regarde en nous racontant l'actualité, et qui se trouve quelque part, dans un lieu appartenant à l'institution médiatique. Le corps du présentateur devient support d'expression et de contact, par le développement des techniques gestuelles; l'espace du plateau s'agrandit, en nous donnant à voir progressivement le mobilier, les salles, les couloirs, la régie, les techniciens, les caméras, les écrans de contrôle, et il est envahi peu à peu par des journalistes spécialisés et par des invités. Ces deux évolutions, celle touchant aux techniques gestuelles du présentateur, d'un côté, et celle de l'expansion de l'espace du plateau et de la multiplication de ses « habitants » de l'autre, sont complémentaires, bien qu'en France la première ait précédé d'un peu la seconde. La substance icônique, le contenu de représentation, de l'image audiovisuelle, devient ainsi matière au service de la structuration progressive d'un dispositif complexe de mise en contact avec le destinataire, espace-temps indiciel où se noue le lien avec le téléspectateur.

6 La tendance profonde qui se fait jour ainsi, à savoir, l'émergence de configurations indicielles de contact, n'est pas propre au journal télévisé : elle apparaît, à des rythmes divers et sous différentes modalités, dans tous les grands genres qui composent aujourd'hui ce qu'on appelle la «télévision grand public » : les magazines d'information, les variétés, les jeux.

7 Un bon exemple est celui d'un genre très important dans l'histoire de la télévision française : les magazines de vulgarisation scientifique. Depuis les émissions classiques à la Pierre Desgraupes, proches du vieux genre du documentaire cinématographique, en passant par la figure de la "table ronde » à la manière de Robert Clark, jusqu'aux émissions des frères Bogdanoff et de Laurent Broomhead dans les années quatre-vingt, la même évolution se fait jour: mise en place progressive de configurations spatiotemporelles où dominent les dimensions du contact. Les émissions de Broomhead sont à cet égard exemplaires : focalisation sur la relation de regard entre un présentateur et le destinataire, multiplication des échanges en direct, travail sur l'architecture du plateau ${ }^{3}$.

8 Au cours de cette évolution, s'est construite la spécificité de la télévision comme média, vis-à-vis du cinéma, et dans le même mouvement, la frontière a été tracée, dans l'univers de l'image audiovisuelle, entre " réalité » et « fiction ». Là où prévaut le contenu icônique de la représentation, c'est du cinéma (même si ça passe à la télévision...) et c'est de la fiction. Dans ce cas, les stratégies énonciatives sont entièrement au service de la narration, elles ne construisent pas de lien avec le destinataire: si, au cinéma, nous « rentrons » dans l'histoire, c'est parce que nous restons irrémédiablement en dehors. Là où les dispositifs de contact dominent sur le contenu de la représentation, c'est du télévisuel et c'est du « réel ». D'où l'importance cruciale du direct à la télévision: le régime en temps réel qu'il implique, "verrouille» le statut non-fictionnel de l'image télévisuelle. 

configurations complexes du contact avec les téléspectateurs, ce qui est en jeu c'est aussi l'institution télévision. On comprend mieux alors pourquoi les journaux télévisés, en particulier celui de vingt heures, qui a l'audience la plus importante, sont devenus, à l'intérieur de la grille de programmes, l'un des lieux privilégiés où une chaîne construit son image de marque. (J'utilise ici la notion de 'marque' dans son sens précis d'entité énonciative imaginaire dans le champ commercial). A l'époque de la télévision monopole d'État, il y avait déjà, bien entendu, une certaine concurrence entre les chaînes, et cette concurrente touchait surtout les journaux télévisés.. Ce qui explique pourquoi, même sous le monopole, les journaux télévisés étaient tous programmés à la même heure. Au temps du monopole, le présentateur du journal était devenu déjà un opérateur de la marque. D'une marque, bien entendu, qui ne s'avouait pas comme telle, dans la mesure où l'audiovisuel restait soumis à la logique « de service public $»^{4}$. chaînes, s'est tout naturellement accentué depuis le début de la privatisation. Des épisodes comme la polémique autour du salaire de Christine Ockrent lorsqu'elle est revenue à Antenne 2 pour présenter le journal de 20 heures, montrent bien que le journal télévisé est au centre des enjeux commerciaux de la télévision. Comme le rappelle aussi la campagne publicitaire récente de la Cinq, construite autour de la figure du présentateur du journal de 20 heures.

15 Les deux principales chaînes, TF1 et Antenne 2, l'une privée, l'autre publique, sont à l'heure actuelle parfaitement installées dans ce que l'on pourrait désigner comme le 
«stade terminal» de cette évolution que j'ai très rapidement évoquée. Si je parle de "stade terminal» c'est parce que l'on peut précisément se demander s'il n'y a pas actuellement l'amorce de quelque chose de nouveau dans l'univers du discours des informations télévisées. Cela ne saurait pas trop nous étonner; ce qui serait surprenant c'est que l'irruption brutale de la logique commerciale dans ce que l'on appelle le "paysage audiovisuel français» puisse avoir lieu sans conséquence sur les fonctionnements discursifs.

Dans la situation extrêmement confuse que connaît l'audiovisuel français aujourd'hui, il est bien risqué de faire des pronostics. La confusion résulte, en bonne partie, du caractère paradoxal de cette entrée de la télévision française dans l'univers de la logique concurrentielle. TF1, la principale chaîne privée, a pris soin d'asseoir son image de marque sur le capital de prestige accumulé lorsqu'elle était une chaîne publique. Et celle qui, de ce point de vue, est la seule véritable chaîne privée, la Cinq, a vu, pour des raisons politiques, son image se construire ailleurs que dans sa propre programmation. A un moment, en effet, où en raison de son faible implantation elle touchait encore une proportion réduite de foyers, beaucoup de Français avaient une image précise, et une opinion tranchée, à propos d'une chaîne qu'ils n'avaient jamais regardée. Entre Berlusconi et Hersant, la Cinq s'est trouvée avec une image de marque construite dans la presse écrite.

Malgré les difficultés qui découlent de cette confusion, essayons, à nos risques et périls, d'identifier quelques symptômes de changement: on les retrouve dans les informations télévisées de la Cinq, seule véritable nouvelle venue dans le champ des chaînes généralistes.

18 Je focalise toujours mes remarques sur le journal de 20 heures, lieu symptomatique par excellence.

19 Dans celui de la Cinq, le plateau a quasiment disparu: après le générique d'ouverture, le présentateur apparaît sur fond noir. Et dans ce noir, le seul élément du mobilier est une sorte de table qui n'en est pas une : une structure qui lui sert de bureau, mais dont la forme reproduit le' 5 'qui est le logotype de la chaîne. Comparativement à TF1 et Antenne 2, le nombre des journalistes spécialisés a été drastiquement réduit: il reste, pour l'essentiel, un éditorialiste politique, et, plus tard dans le journal, un invité. L'espace est comparativement vide.

Dans les génériques d'ouverture et de fermeture du journal, la différence entre le journal de la Cinq et ceux des deux autres grandes chaînes, TF1 et Antenne 2, est saisissante : tandis que celles-ci montrent, comme il est devenu la norme, le plateau avec ses composantes techniques, les génériques de la Cinq montrent la planète et un satellite qui tourne autour. Comme l'image même du présentateur, qui dans le générique de fermeture, se mettra à faire le tour de la terre avant de disparaître dans les profondeurs de l'espace. Le peu qui reste du plateau est vide, et le présentateur flotte dans les espaces inter-planétaires : il est partout et nulle part.

21 Dans la construction même du journal, le privilège est accordé aux images événementielles, avec de longs reportages en extérieurs, articulés par un discours descriptif bref et dépouillé.

Sommes-nous revenus aux origines de la télévision ? S'agit-il d'un retour au « journalisme de reportage» du bon vieux temps des années soixante? Ces symptômes, dont l'importance est d'autant plus grande que leur émergence, répond aux lois strictes de la concurrence, on pourrait sans doute les interpréter comme un retour du refoulé 
(l'énoncé), après tant d'années de mise en spectacle des stratégies énonciatives du contact.

Une telle interprétation serait, sans doute, un peu hâtive. Car l'histoire, nous le savons, ne fait jamais vraiment des retours en arrière, et celle des médias en particulier, n'a que faire des revenants. La période de l'innocence du «présentateur ventriloque», dont la crédibilité était entièrement en dehors de lui-même, est définitivement révolue.

Mais peut-être que l'heure est venue du déclin relatif des stratégies énonciatives affichées comme médiation interprétative (et protectrice) entre le destinataire et l'actualité du monde. Déclin relatif : ces stratégies sont toujours là, elles animent les journaux télévisés des deux chaînes leaders en France : l'une publique, l'autre qui fait tout pour que l'on oublie qu'elle ne l'est plus. Mais l'irruption de la logique du marché rend possible aujourd'hui l'émergence d'une offre légèrement différente, un peu en décalage: un journal télévisé construisant un destinataire qui manifestement cherche essentiellement à être mis en contact avec les événements, pour en faire son propre jugement (il y est invité, tous les soirs, à travers le Minitel). Ce qui est, vous me direz, une stratégie énonciative comme une autre. Bien entendu. Mais qui est très différente des autress, et très différente aussi de celle qui caractérisait le dispositif primitif du présentateur ventriloque : par la bouche de celui-ci, passait le discours vrai de l'information. Or, la médiatisation des sociétés industrielles a comme effet premier la mise en évidence des stratégies énonciatives : mise en équivalence des discours, qui est l'un des mécanismes fondamentaux de la démocratie. Dans ce contexte de médiatisation qui est le nôtre aujourd'hui, radicalement différent de celui des premières années de la télévision, il n'y a plus de "discours vrai ». Les symptômes dont nous parlons doivent donc être replacés dans le cadre de l'évolution socioculturelle, et en particulier dans le cadre des transformations de l'individualisme. Si retour de l'énoncé il y a, il préannonce peut-être des modalités discursives qui transferent au destinataire une partie des tâches auparavant dévolues à l'énonciateur : celles de l'interprétation.

\section{BIBLIOGRAPHIE}

Brusini, H. et James, F., (1982), Voir la vérité. Le journalisme de télévision, Paris, Presses Universitaires de France.

Véron, É., (1983), « Il est là, je le vois, il me parle », Communications, 38, pp. 98-120.

\section{NOTES}

1. H. Brusini et F. James, Voir la vérité. Le journalisme de télévision, Paris, Presses Universitaires de France.

2. É. Véron, « Il est là, je le vois, il me parle », Communications, 38, 1983, pp. 98-120. 
3. On trouvera une analyse des stratégies énonciatives dans les magazines de vulgarisation scientifique dans: É. Fouquier et É. Véron, Les spectacles scientifiques télévisés. Figures de la production et de la réception, Paris, Ministère de la Culture-la Documentation Française, 1985.

4. A plusieurs reprises, pendant cette longue histoire de la télévision de service public en France, le changement de décor du journal télévisé de 20 heures a coïncidé avec un changement de gouvernement : la restructuration de l'architecture du plateau des informations était devenue le symbole d'un changement de politique audiovisuelle.

\section{RÉSUMÉS}

La télévision, en tant que media audiovisuel, allie le son et l'image. L'auteur se propose d'y analyser leurs rapports à travers l'étude du langage audiovisuel et, plus spécifiquement, des modalités de discours observées dans l'exercice du journal télévisé. Ces modalités ne sont pas seulement d'ordre linguistique : l'auteur analyse les espaces énonciatifs, les espaces discursifs, c'est-à-dire l'articulation entre les discours journalistiques et les images qui lui sont associées.

\section{INDEX}

Keywords : television news, journalism, audiovisual, enunciative strategy, speech, semiology

Mots-clés : journal télévisé, journalisme, audiovisuel, stratégie énonciative, discours, sémiologie

\section{AUTEUR}

\section{ELISÉO VÉRON}

Éliséo Véron, Université de Paris I 